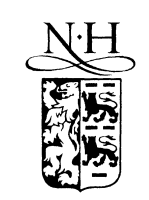

ELSEVIER

\title{
Density of vibrational states of fractal aggregates: scalar interactions
}

\author{
Romain Thouy ${ }^{\mathrm{a}, *}$, Rémi Jullien ${ }^{\mathrm{a}}$, Claude Benoit ${ }^{\mathrm{b}}$ \\ ${ }^{a}$ Laboratoire des Verres, Université Montpellier II, Place Eugène Bataillon, 34095 Montpellier, France \\ ${ }^{\mathrm{b}}$ Groupe de Dynamique des Phases Condensées, Université Montpellier II, Place Eugène Bataillon, 34095 Montpellier, France
}

\begin{abstract}
Using a hierarchical computer algorithm, we have built on- and off-lattice disordered fractal aggregates made of identical tangent spheres, whose fractal dimension can be varied from 1 to an upper limit of about 2.5. We have computed the density of vibrational states for different fractal dimensions, using a fast spectral moments method, for the case of scalar interactions. In this approach, we used two link models which take into account the presence or not of loops in the structure. The spectral dimension as a function of the fractal dimension is discussed. We study the connectivity dimension, $\hat{d}$, and the mean coordination number, $z$, to get more information on the connections within the structures. We give some comparisons with experiments on real materials. (c) 1998 Published by Elsevier Science B.V. All rights reserved.
\end{abstract}

\section{Introduction}

It is well known that the vibrational density of states (DOS) varies as a power, $\left(d_{\mathrm{s}}-1\right)$, of the frequency above a phonon to fraction crossover frequency, $\omega_{\mathrm{c}}$, where $d_{\mathrm{s}}$ is the spectral dimension $[1,2]$. Until now, most of the studies about fraction excitations [3] in fractal structures have used either percolating networks, the diffusion limited clusterclostet aggregation (DLCA) model [4] or a few deterministic fractal structures (such as Sierpinski gasket [5]). In each case, the fractal dimension was fixed and could not be changed. To study physical properties of fractal aggregates, as a function of their fractal dimension, we have developed an aggregation model $[6,7]$ which is able to build frac-

\footnotetext{
${ }^{*}$ Corresponding author. Tel.: 334 67144677; fax: 334 67143498; e-mail: thouy@1dv.univ-montp2.fr.
}

tal aggregates, with a fractal dimension that can be changed. We use this model to systematically investigate the behavior of the DOS as a function of the fractal dimension. We computed the DOS of these aggregates for the case of scalar interactions, using a spectral moments method (SMM). It is well known that the spectral dimension, $d_{\mathrm{s}}$, is not directly related to the fractal dimension, $D$, but rather to the connectivity of the particles. Therefore, we have also calculated the spreading dimension, $\hat{d}$, which is another intrinsic parameter of the fractal structure affected by the connectivity, and the mean number of bonds per particle, $z$, in order to relate them to the behavior of $d_{\mathrm{s}}$.

In Section 2, we give a brief summary of the model employed in this study, and we explain our two model links for particle interactions. Then, in Section 3, we recall the main principle of the SMM used to compute the DOS, and we explain how to compute $\hat{d}$ and $z$. Results of $d_{\mathrm{s}}, \hat{d}$ and 
$z$ obtained for several dimension, $D$, are presented in Section 4 , and a comparison with experiments on aerogels (for $d_{\mathrm{s}}$ ) is discussed in Section 5. We conclude in Section 6.

\section{The aggregation and dynamical models}

Different models have been introduced to describe aggregation phenomena, such as those observed in experiments on colloids and aerosols. The original DLCA model [8,9], which is appropriate for the quick aggregation of screened colloidal particles, considers aggregates that undergo Brownian motion and stick irreversibly when they come into contact. The ballistic model (BCA) $[10,11]$, which is more appropriate for aerosols in the molecular regime, considers independent random straight-line trajectories for the clusters. The chemically limited aggregation model (CLCA) $[12,13]$, also called 'reaction-limited' aggregation $[14,15]$, which applies to the slower aggregation of partially screened colloids, considers that aggregates should collide many times before they stick. These three models lead to aggregates of increasing fractal dimensions, $D$, of $1.78,1.98,2.05$ in dimension $d=3$, for DLCA, BCA, CLCA, respectively.

The variable-D model is generalization of these previous models. It is based on a hierarchical procedure introduced by Botet and collaborators $[16,17]$ and has the great advantage of containing the fractal dimension as an input parameter. This model allows samples of fractal aggregates with various fractal dimensions ranging from 1 to 2.5 (for $d=3$ ) to be built.

The procedure starts with a collection of $2^{n}$ particles. These particles are grouped into pairs. Each pair generates an aggregate of two particles so that, at iteration $p=1$, one obtains $2^{n-1}$ dimers. The dimers are grouped into pairs that generate tetramers and so on. At iteration $p$ one obtains $2^{n-p}$ aggregates containing $N=2^{p}$ particles each. The procedure stops at iteration $p=n$, where a final aggregate of $2^{n}$ particles is obtained. An aggregate of the next generation, containing $2 \mathrm{~N}$ particles, is obtained by sticking two aggregates of the previous generation, containing both $N$ par- ticles, in such a way that the distance, $\Delta$, between their centers of mass should satisfy at best the condition (see Ref. [6])

$\Delta^{2}=k^{2} \frac{R_{N 1}^{2}+R_{N 2}^{2}}{2}+1$,

where $R_{N 1}^{2}$ and $R_{N 2}^{2}$ are the mean square radii of gyration of the two aggregates of the previous generation, and where $k$ is related to the desired fractal dimension, $D$, by

$k^{2}=4\left(4^{1 / D}-1\right)$.

The corrective term +1 in Eq. (1), which becomes negligible for large aggregates, is introduced to satisfy exactly $R_{N}^{2}=\left(N^{2}-1\right) / 12$ for $D=1$ (linear chain of $N$ tangent particles).

To summarize, given $D$, which here becomes an input parameter, one can calculate $k$ by Eq. (2) and if one can manage to insure (1) during the sticking of two aggregates of $N$ particles, one should be able to build fractal aggregates of desired fractal dimension. The main difference between this model and previous models (such as DLCA, BCA and CLCA) is that the natural distribution of the penetration parameter, $\Delta$, which depends on the physical aggregation process, is here replaced by a Dirac peak. Thus, in addition to the hierarchical approximation, which is to neglect the polydispersity of aggregates, we introduce another approximation which is to neglect the polydispersity of their penetration distances.

In the on-lattice version of the model, the particle centers are restricted to sites of a $d$-dimensional hypercubic lattice with unit parameter, while in the off-lattice version, they can vary continuously. The off-lattice version has the advantage that Eq. (1) is rigorously satisfied for all $N$. In both cases, the resulting fractal dimension has been calculated and has been shown to be equal to the input $D$, unless it is not larger than a $d$-dependent upper limit which has been determinated for $d$ between 2 and 6. For $d=3$ the limit is $D \simeq 2.55$. The fact that one cannot reach fractal dimensions as large as $D=d$ is due to geometrical frustrations induced by disorder: our hierarchical method introduces natural surface roughness while, to reach $D=d$, one needs a smooth surface. A way to avoid this limitation, while preserving randomness, would 
be to introduce some cluster-size polydispersity in a controlled way.

We show in Fig. 1, pictures of three-dimensional on-lattice aggregates, with sizes up to 16384 particles, and fractal dimension $D=1.5$ (a), $D=2.0($ b) and $D=2.5$ (c).

For the study of the spectral dimension, we consider, in the on-lattice case, two kinds of link rules, called: (i) ALLINKS: links to all first neighbors are considered; (ii) ONELINK: only the real links created by the aggregation model are considered. In the latter case, there are no loops, i.e. there are $(N-1)$ links for an aggregate of $N$ particles, as in the off-lattice case. Fig. 2, shows how we stick together two aggregates of four particles (one is represented by open circles, and the other by filled circles), following ONELINK and ALLINKS rules. We have drawn all bonds with springs.

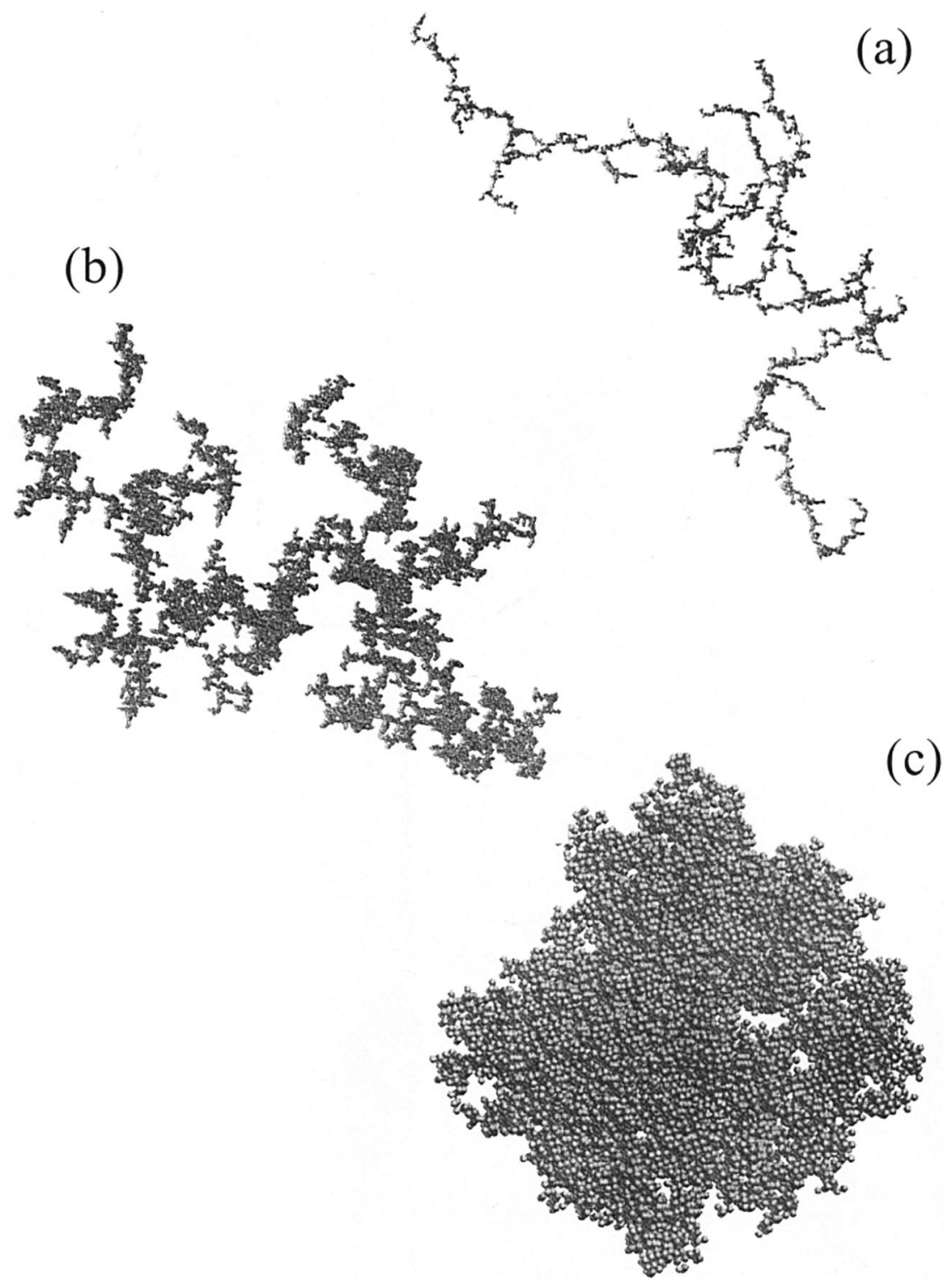

Fig. 1. On-lattice aggregates containing 16384 particles each, with fractal dimension $D=1.5$ (a), $D=2.0$ (b) and $D=2.5$ (c). 


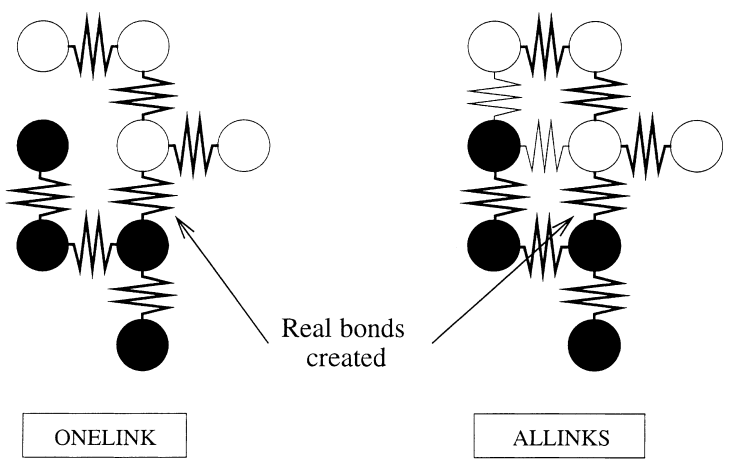

Fig. 2. Final aggregate with eight particles $(d=2)$, obtained by sticking two aggregates of four particles each (black and white circles), where bonds are represented by springs. In the case ALLINKS, there are nine bonds, in the case ONELINK, there are seven bonds.

There is some interest in studying these two cases. The ONELINK case is very close to what would be obtained in a real cluster-cluster process with 'rigid' clusters while the ALLINKS case would correspond to some degree of 'restructuring' when extra bonds can be formed (due to cluster flexibility or the free rotations of bonds). Since some loops are created in the ALLINKS case (which are not present in the ONELINK case), it is interesting to know if they are relevant in modifying the properties of the spectral density.

We now assume that particles are connected by springs. The displacements of particles are represented by scalars. Then, the set of equation of motion for the site $i$ is given by

$m \ddot{U}_{i}=-\sum_{j} \gamma_{i j} k U_{j}=-\sum_{j} k_{i j} U_{j}$

with

$k_{i j}=-\sum_{j \neq i} \gamma_{i j} k$.

$U_{i}$ is the scalar displacement of the site $i, k_{i j}$ are force constants between particles $i$ and $j, \gamma_{i j}$ are coefficients depending on the link rules. For the ALLINKS model, $\gamma_{i j}=1$, if $i$ and $j$ are occupied, while, with the ONELINK model, $\gamma_{i j}=1$ only if a real link has been created between particles $i$ and $j$ by the aggregation model (Fig. 2). Here, we have used $m=1$ and $k=1$.

\section{Numerical method}

We have used the spectral moments method (SMM) $[18,19]$ which is a powerful tool to study the dynamical properties of harmonic systems. This method has been applied to the study of fractal structures, percolating networks and silica aerogels $[24,25]$. The DOS is given by

$g(\omega)=\sum_{j} \delta\left(\omega-\omega_{j}\right)$

where $\omega_{j}$ is the frequency of the $j$ th eigenmode. The eigenvalue, $\lambda_{j}$, of the dynamic matrix, $D$, being equal to the square of the frequency. It is more convenient to work with $u=\omega^{2}$ and $\lambda_{j}=\omega_{j}^{2}$, and compute

$G(u)=\sum_{j} \delta\left(u-\lambda_{j}\right)$

the squared frequency distribution. We do not describe, the principles of the SMM: for more details see Ref. $[18,19]$. This method gives very good results with very large systems. If the system is small $\left(N \propto 10^{3}\right)$, some difficulties appear when computing the slope of the log-log plot of the density of states. We find fluctuations which arise from the presence of small gaps in the spectrum. To obtain more accurate results, we have directly computed the integrated density of states (IDOS). We compute the function $H(u)$, such that

$H(u)=\int_{0}^{u} G\left(u^{\prime}\right) \mathrm{d} u^{\prime}$,

so that from Eq. (7)

$H(u) \propto u^{d_{s} / 2}$.

To test the accuracy of the SMM, we have previously calculated the DOS for small aggregates, and compared with results obtained by a direct diagonalization (which gives exact results), for the same aggregates. As the computation of diagonalization is limited to matrices no larger than about $3000 \times 3000$, we have used SMM on aggregates of 2048 particles, to obtain a good comparison. All these results have been published in Ref. [20]. For the other calculations, we have worked on samples with 8192 particles, for aggregates built 
on-lattice. In this work, also we add results for offlattice aggregates of size up to 32768 particles, where such aggregates correspond to ONELINK case calculations of on-lattice aggregates, in terms of connectivity.

To get more information on the connectivity of our systems, we have calculated the spreading dimension, $\hat{d}$ (or chemical dimension) [21-23], which is defined as follows: starting from a particle of the aggregate, one covers a distance, $L$, following a walk entirely contained in the cluster. There are many ways to do so. We count all the visited particles, whatever the walk. This number varies as

$$
n(L) \propto L^{\hat{d}},
$$

where $\hat{d}$ is called the spreading dimension. This exponent only depends on the precise ways the particles are connected, but not of their repartition in space. The conventional order the different exponents follow is written as

$d_{\mathrm{s}} \leqslant \hat{d} \leqslant D$.

Due to the special behavior found for $d_{\mathrm{s}}$ and $\hat{d}$, we have calculated the mean number of coordination per particle, $z$. For off-lattice clusters (and onlattice one, in ONELINK case) of $N$ particles,

$z=2-\frac{2}{N}$

but there was no trivial value for on-lattice aggregates in the ALLINKS case. The coordination number, $z$, is not directly relatable to $\hat{d}$ or $d_{\mathrm{s}}$, but it could help to understand their behavior.

\section{Results}

We consider now the SMM results, reported in Fig. 3. These results concern clusters built on-lattice (empty symbols) and off-lattice (filled square). The results are averaged over five samples of $N=8192$ particles, for on-lattice clusters, and over four samples of $N=32768$ particles, for off-lattice clusters. As explained above, we have plotted the ONELINK (open triangle) and ALLINKS (empty triangle) results. In the two cases (ONELINK and ALLINKS) $d_{\mathrm{s}}$ increases monotically with $D$, and, for larger $D$, the $d_{\text {s }}$ are larger for the ALLINKS than for the ONELINK model. In both ALLINKS

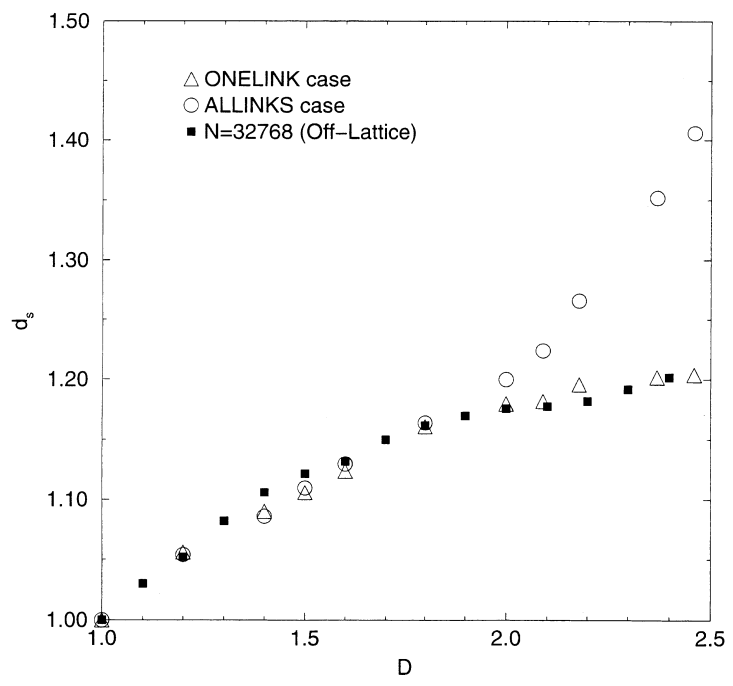

Fig. 3. Spectral dimension calculated by the SMM, for aggregates with 8192 particles, averaged for five samples (empty symbols), and for aggregates built off-lattice, with size up to 32768 particles, averaged over four samples (filled square). We present the two cases: ALLINKS (circle) and ONELINK (triangle). Experimental points are indicated by diamonds, respectively for basic aerogels $(D=1.8)$ and for neutral aerogels $(D=2.3)$.

and ONELINK models, the results for $D<1.8$ are almost superimposed. For $D>1.8$, they separate: while $d_{\mathrm{s}}$ becomes almost $D$-independent $\left(d_{\mathrm{s}} \simeq 1.20\right)$ in the ONELINK case, it increases in the ALLINKS case. This last result can be understood since $d_{\mathrm{s}}$ should tend to 3 in the ALLINKS case when $D \rightarrow 3$, while there is no obvious $D=3$ limit in the ONELINK case (the structure stays like a branched polymer in that limit).

One can see that results obtained for off-lattice clusters are close to the one obtained for on-lattice clusters, in the ONELINK case. This similarity is due to the same connectivity properties of these two kinds of clusters, generated with the same construction laws. The lattice has no effect on the behavior of $d_{\mathrm{s}}$.

The curves for ONELINK and ALLINKS cases separate for a fractal dimension, $D$, of about 1.8. In order to relate this observation with the connectivity of the aggregates, we have plotted in Fig. 4, the spreading dimension, $\hat{d}$, as a function of the fractal dimension, $D$, for the ONELINK and ALLINKS cases, averaged over the same samples. $\hat{d}$ increases with $D$, in the two cases, but more rapidly in the 


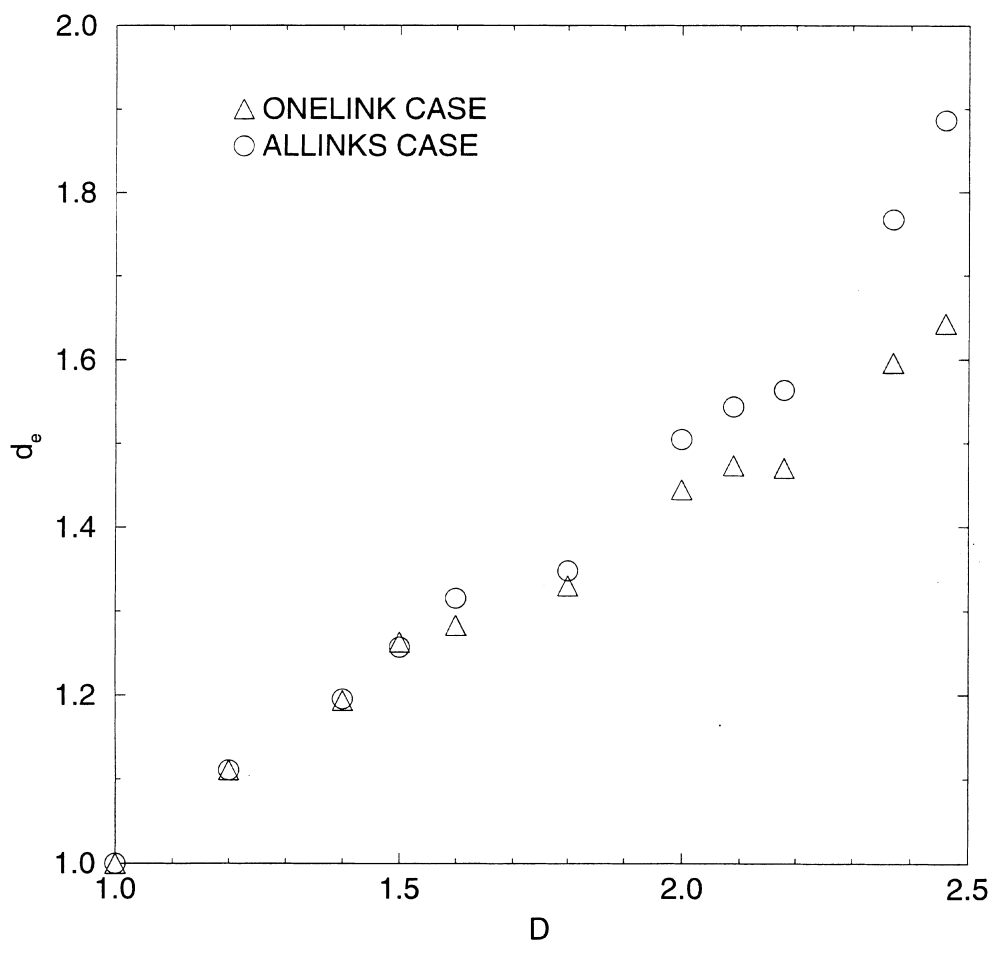

Fig. 4. Plot of $d$ as a function of the fractal dimension, $D$, for on-lattice samples, for two cases ONELINK (empty triangle) and ALLINKS (empty circle), averaged over five samples.

ALLINKS case: this difference is due to the presence of loops, in the structure, which start to be relevant when $D=1.8$ and affect both the spectral dimension, and the spreading dimension.

A reason why the number of loops increased in the structure could be due to the mean connectivity of the particles. We have plotted, in Fig. 5, the mean number of coordination, $z$, as a function of the fractal dimension, $D$, calculated for the same samples (empty circle), for the two models links. We have also reported, on the same graph, values obtained for bigger samples, with sizes up to 16384 and 32768 particles (without average). As expected in the ONELINK case, $z$ is constant, close to 2, whatever the fractal dimension. But in the ALLINKS case, $z$ has a nontrivial behavior, close to 2.1 , for $D \leqslant 1.7$, and close to 2.5 for $2.0 \leqslant D$. There is a discontinuity between the two regimes, around $D=1.8$. We verify that these exponents are in the following order

$d_{\mathrm{s}} \leqslant \hat{d} \leqslant D$ as conjectured by Rammal and his collaborators [23].

\section{Discussion}

At this stage it is interesting to compare the present theoretical results with the existing experiments, especially in the case of aerogels, where the existence of fractons has been demonstrated. The spectral dimension of aerogels has been estimated from Brillouin scattering experiments giving $d_{\mathrm{s}} \simeq 1.1$ (see Fig. 3, empty diamond) when they are prepared with a basic catalyzer [26] and $d_{\mathrm{s}} \simeq 1.3$ (see Fig. 3, empty diamond) without a catalyzer [27]. The spectral dimension of basic aerogels is in good agreement with the present work if one understands that their fractal dimension is $D=1.8$ [28], corresponding to $d_{\mathrm{s}} \simeq 1.15$ in both ONELINK and ALLINKS cases. Moreover, it is known that small angle neutron scattering experiments are very well explained by 


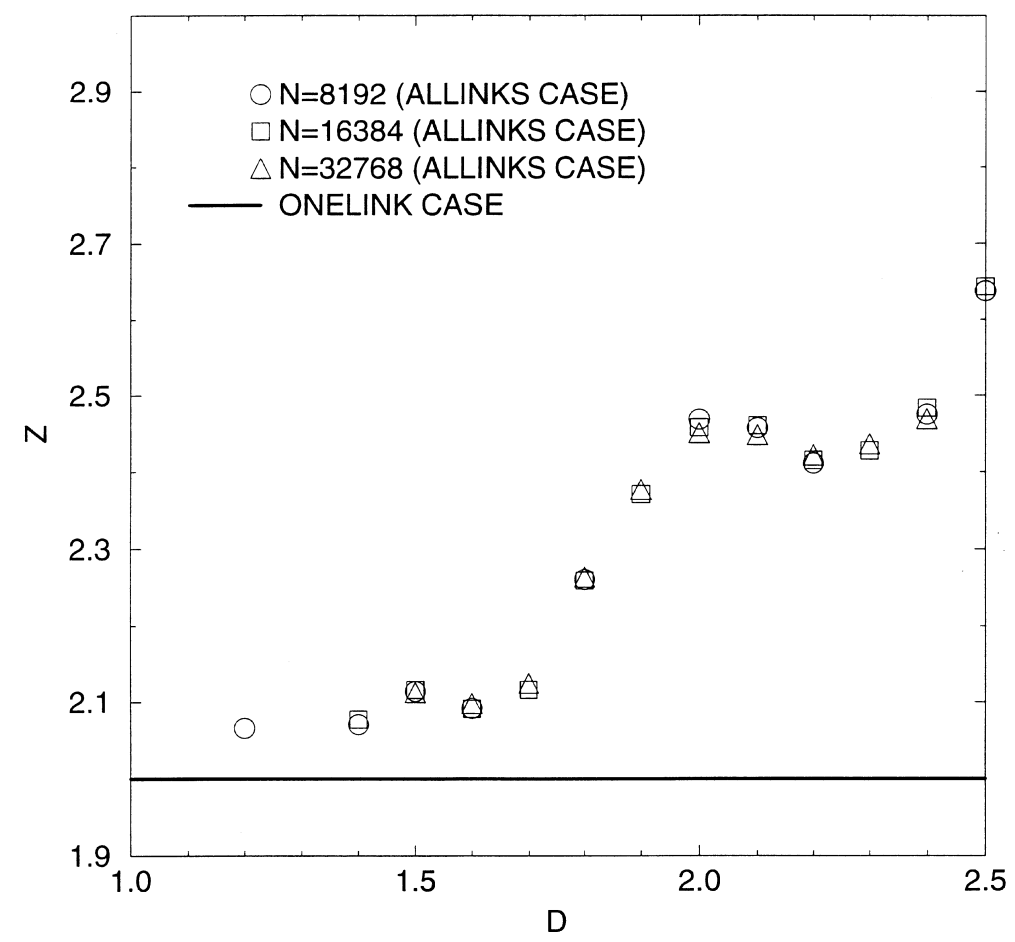

Fig. 5. Plot of $z$ the mean coordination number, versus $D$, the fractal dimension, for the ONELINK (bold line) and ALLINKS (symbols) cases. Symbols represent different sizes of clusters: $N=8192$ particles (circle), $N=16384$ particles (circle) and $N=32768$ particles (circle).

numerical simulations based on the DLCA model [29] which is a model very close to the variable- $D$ model for $D=1.8$. To discuss the case of neutral aerogels, one should consider that a fractal dimension of $D=2.3$ gives $d_{\mathrm{s}} \simeq 1.2$ in the ONELINK case and $d_{\mathrm{s}} \simeq 1.3$ in the ALLINKS case. Again, this seems to be in agreement with the experiment if one takes into account that the structure of neutral aerogels is polymeric and certainly has many more loops than the structure of a basic aerogel [30]. The behavior of $\hat{d}$ is the same as for $d_{\mathrm{s}}$ : until $D=1.8$, the number of loops is too small to modify the DOS. In the same range of $D, z$ is constant (close to 2.1), and confirms our observation.

\section{Conclusion}

In this paper, we have reported an explicit variation of the spectral dimension, $d_{\mathrm{s}}$, and of the spreading dimension, $\hat{d}$, as a function of the fractal dimension for a series of aggregates of tunable fractal dimension. The results have been compared with experiments performed on fractal aerogels. We have shown numerically the existence of a threshold about 1.8 for the fractal dimension, above which the existence of internal loops in the structure affects longitudes of $d_{\mathrm{s}}$ and $\hat{d}$. This threshold corresponds to the fractal dimension above which the mean coordination number becomes larger than 2. It is interesting to notice that this threshold value is larger than the 'transparency' criterium $D=d / 2$ (1.5 in three dimensions) below which it is known that loops are irrelevant.

\section{Acknowledgements}

Part of the numerical calculations were done at CNUSC (Centre National Universitaire Sud de Calcul), Montpellier, France. 


\section{References}

[1] S. Alexander, R. Orbach, J. Phys. Lett. 43 (1982) L625.

[2] R. Rammal, G. Toulouse, J. Phys. (Paris) 44 (1983) L13.

[3] T. Nakayama, K. Yakubo, R.L. Orbach, Rev. Modern Phys. 66 (1994) 381.

[4] A. Rahmani, C. Benoit, R. Jullien, G. Poussigue, A. Sakout, J. Phys. CM 8 (1996) 5555.

[5] C. Benoit, G. Poussigue, A. Assaf, J. Phys. CM 4 (1992) 3153.

[6] R. Thouy, R. Jullien, J. Phys. A 27 (1994) 2953.

[7] R. Thouy, R. Jullien, J. Phys. I (Paris) 6 (1996) 1365.

[8] P. Meakin, Phys. Rev. Lett. 51 (1983) 1119.

[9] M. Kolb, R. Botet, R. Jullien, Phys. Rev. Lett. 51 (1983) 1123.

[10] R. Ball, R. Jullien, J. Phys. (Paris) Lett. 45 (1984) L1031.

[11] R. Jullien, J. Phys. A 17 (1984) L771.

[12] R. Jullien, M. Kolb, J. Phys. A 17 (1984) L639.

[13] M. Kolb, R. Jullien, J. Phys. (Paris) Lett. 45 (1984) L977.

[14] W. Brown, R. Ball, J. Phys. A 18 (1985) L517.

[15] F. Family, P. Meakin, T. Vicsek, J. Chem. Phys. 83 (1985) 4144.

[16] R. Botet, R. Jullien, M. Kolb, J. Phys. A 17 (1984) L75.
[17] P. Meakin, J. Colloid Interf. Sci. 102 (1984) 491.

[18] C. Benoit, E. Royer, G. Poussigue, J. Phys. 4 (1992) 3125.

[19] C. Benoit, J. Phys. 6 (1994) 3137.

[20] R. Thouy, R. Jullien, C. Benoit, J. Phys. 7 (1995) 9703.

[21] J. Vannimenus, J.P. Nadal, H. Martin, J. Phys. A 17 (1984) L35.

[22] S. Havlin, R. Nossal, J. Phys. A 17 (1984) L427.

[23] R. Rammal, J.C. Angles d'Auriac, A. Benoit, J. Phys. A 17 (1984) L491.

[24] A. Rahmani, C. Benoit, E. Royer-Vilanova, G. Poussigue, J. Phys. 5 (1993) 7941.

[25] A. Rahmani, C. Benoit, G. Poussigue, J. Phys. 6 (1994) 1483.

[26] E. Anglaret, A. Hasmy, E. Courtens, J. Pelous, R. Vacher, Europhys. Lett. 28 (1994) 591.

[27] E. Courtens, R. Vacher, J. Pelous, T. Woigner, Europhys. Lett. 6 (1988) 245.

[28] R. Vacher, T. Woigner, J. Phalippou, J. Pelous, E. Courtens, J. Non-Cryst. Solids 106 (1988) 161.

[29] A. Hasmy, S. Anglaret, M. Foret, J. Pelous, R. Jullien, Phys. Rev. B 50 (1994) 6006.

[30] R. Jullien, A. Hasmy, Phys. Rev. Lett. 74 (1995) 4003. 